$$
(10 N-31647--4
$$

LA-UR-81-2410

TITLE: INTE" MEDIATE-ENERGY PROTON AND LIGHT-ION SCATTERINÒ

AUTHOR(S): J. M. Moss

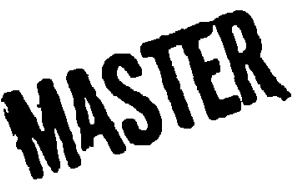

SURMITTED TO: Versailles Conference, Versailles, France, July 6-10, 1981

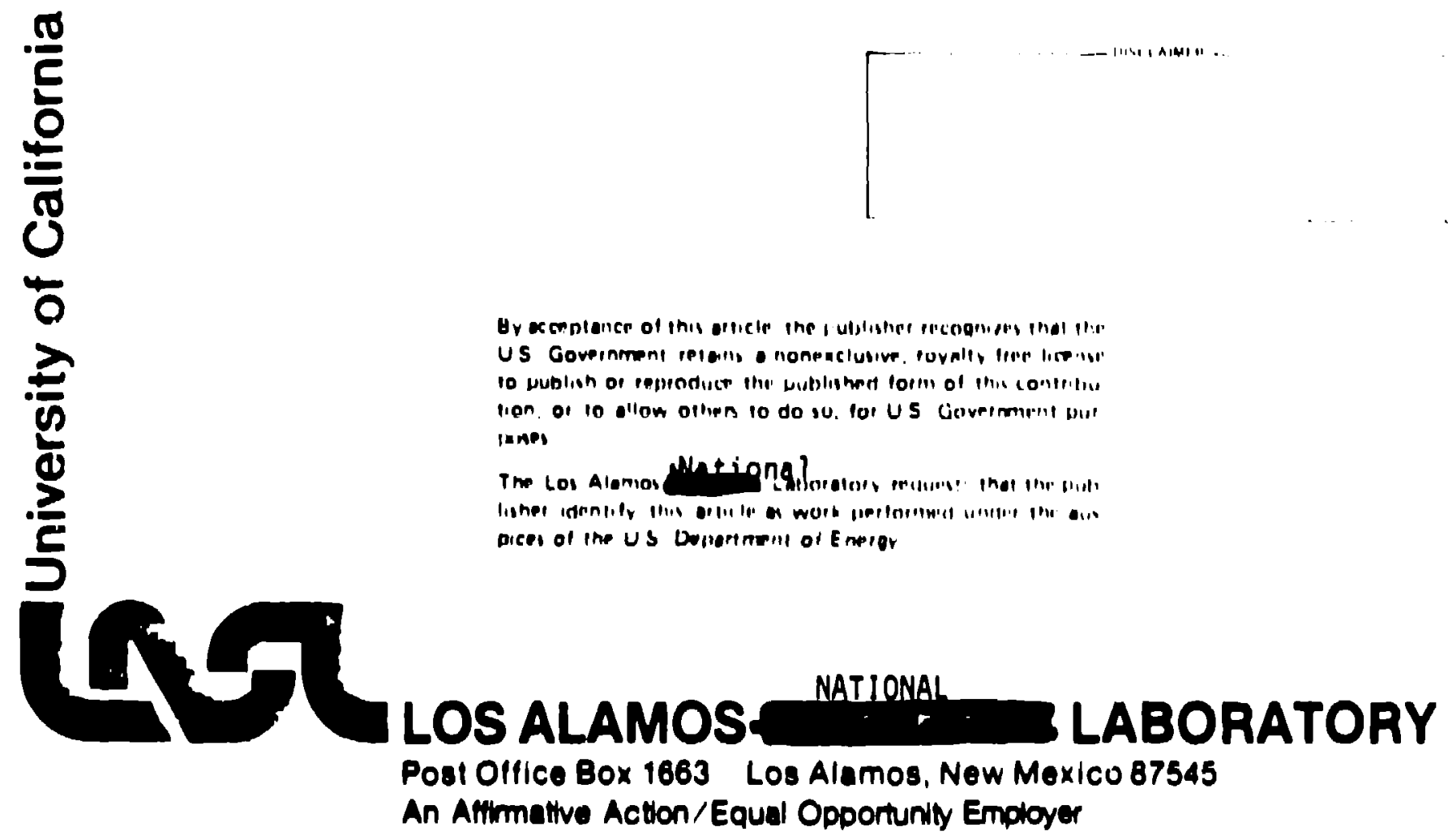

Form No. 250 ค 


\section{INTERMEDIATE ENERGY PROTON AND LIGHT-ION SCATTERING}

J. M. MOSS

Los Alamos National Laboratory

Los Alamos, New Mexico, U.S.A.

A review is presented of recent (1979-81) developments in the field of interinediate-energy protcn and light-ion scattering from nus le theoretical and calculational techniques of particular interest to experimentalists are discussed. Emphasis is placed on topics in nuclear structure physics - giant resonances, pion-condensation precursor phenomena, and polarization transfer (spin-flip) experiments - where intermediate energy proton and light-ion scattering has made new and unique contributions.

\section{Introduction}

The general features of high-energy proton scattering in the study of nuc lear-structure physics have been emphasized in several ICOH.EPANS talks in the past ${ }^{1}$, hence 1 will concentrate on the new developments in this subfield, particularly those that have appeared since the 1979 vancouver ionference. This list (tahle 1) appears as a collection of disoarate subjerts

Table 1 - Outiine of Subjects Covered

1. Int roduction

2. iN-N Interaction and Recent Theoretical Developments $\mathrm{N}$-iv linteraction

DWIA description of inelastic scattering

Dot.a-to-data onal; ses

3. Nuciear Structure and Reaction Giart Resonarices

Delta-isodar-hole configurations

Pion-condensationi precursors

Measurement of the $Q$ parameter

4. Summary

Spin-flip measurements

having no central unifying theine. Although there is some underlyinu unily not apparent in the titles, the first impression is essentially correct. After all, proton and light-ion scattering is a tool when allows us to investigate certain properties of nuclet and nuclear interactions, not an end in itseif. A very important point, moreover, which has been emphasized recently, ${ }^{2}$ is that the most effective use of this tool is of ten in conjunction with other tools, spec if ically the scattering of mesonic and electromagnetic probes, in the pursisit of a common goal in nuclear structure physics.

A notable absence in the list in table 1 is the subject of neutron rajit. This omission ret lects my view that the field of elastic scattering is now at the stage of asking. "Just how reliable are these calculations, anyway?"I 1t. also ref lects my predjudice that neutron radil have been talked abcut enough recent ly."

\section{The $N-N$ interact ton and Recent Thzoret tcal Developments}

Fundamental to the use of proton and light-ion scattering as a probe of nuclear structure are the theories employeu to describe the reaction. The founjattons of the commonly used inultiple scattering theortes were laid down over 20 years ago." New developments cont inue to occur and have been reviewed recently." In this section I will "liscuss a few developments fromi 
ar. experimentalist's point of view, which are very important in terms of understanding and interpreting experimental data.

A crucial ingredient in the theoretical discription of nucleon scattering at high energies is an accurate knowledge of the free $\mathrm{N}-\mathrm{N}$ interaction. Phase-shift analyses for energies at and below $500 \mathrm{MeV}$ have been available for some time. Recently the data base from $500 \mathrm{MeV}$ to $800 \mathrm{MeV}$ has been considerably augmented, with the result that phase-shift analyses with considerably fewer ambigulties are now available in this range." It should be emphasized, rowever, that even for energies where the phase shifts are "well-known", the N-N scattering amplitudes may not be very well determined in the range of momentum transfer where most $\mathrm{N}$-nucleus experiments are performed.

Of considerable importance are theoretical and calculational developments which aid experimentalists in planning experiments and in unders anding their data. I will discuss two such developments; the work of Love and Franey ${ }^{3}$ who have constructed a general distorted-wave impulse approximation (DWIA) code for inelastic scattering, and the work of the Penn group'o who have formulated a simple and elegant "data-to-data" version of multiple-scattering theory.

Figure I snows schematically the procedure employed for DWIA calculations. The cructal step is to represent the N-N Scattering amplitudes in terms of a sum of Yukawa potentials which can then be used in ant1-symmetrized coordinate-space DWIA calculations employing nearly any set of wave functions one desires to test. Such calculations include effects from all the terms in the Q-space N-N scattering amplitudes.

Since the work of Love and franey one has a more global view of the energy dependence of the various pleces of the N-N interaction. Figure 2 shows the energy dependence of the strengths of the four spin and isospin dependent pleces of the central interactiun at $a=0$. It is immediately clear that in the range near $E_{p}$ - $800 \mathrm{MeV}$ scalar-isoscalar transitions will predominate, while the range from 200 to $400 \mathrm{MeV}$ is opt imum for the study of spin-flip transitions with minimum interference from scalar-isoscalar reactions.

The validity of the DWIA for hadrons becomes more dubious the lower the beam energy. For protons, the range $E_{p}=150 \mathrm{MeV}$ might seem +ou low, and indeed there have been some notable fallures of the Díla nere. 11,12 An approach that seems very promising at lower energies is a simple correction of the effective $N-N$ interaction in the form of density dependence which is taken from the local density approximation. 12 The improvement in the quality of fits to both cross sections and analyzing powers is remarkable for the isoscalar transitions to which tints ccrection has been applied.

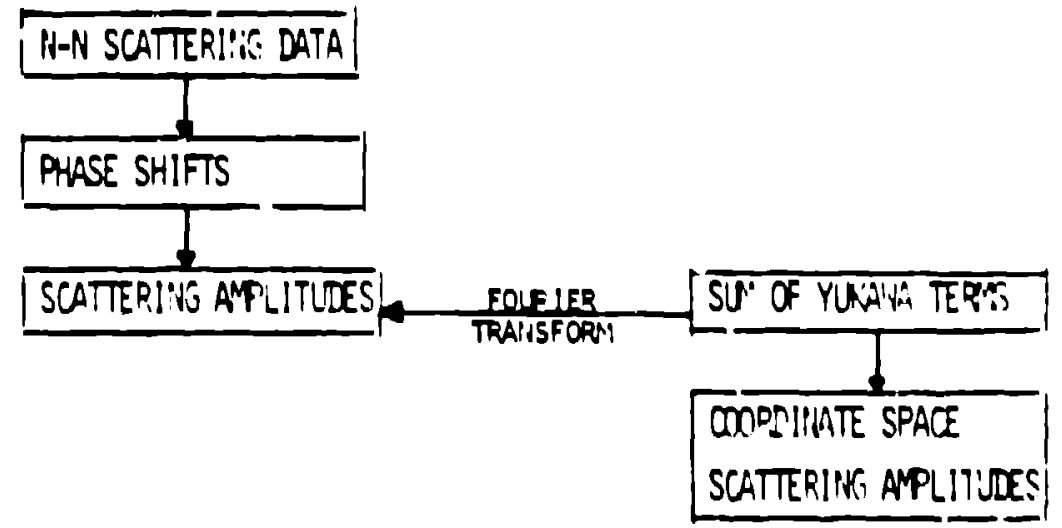

Fig. I senematic procedure for obtaining coordinate space scattering anmplitudes for use in DWIA calculations. The cruclal step is a fit of Fourler-transformed Yukawa terms to the 4 -space $N-N$ scattering amplitudes. 


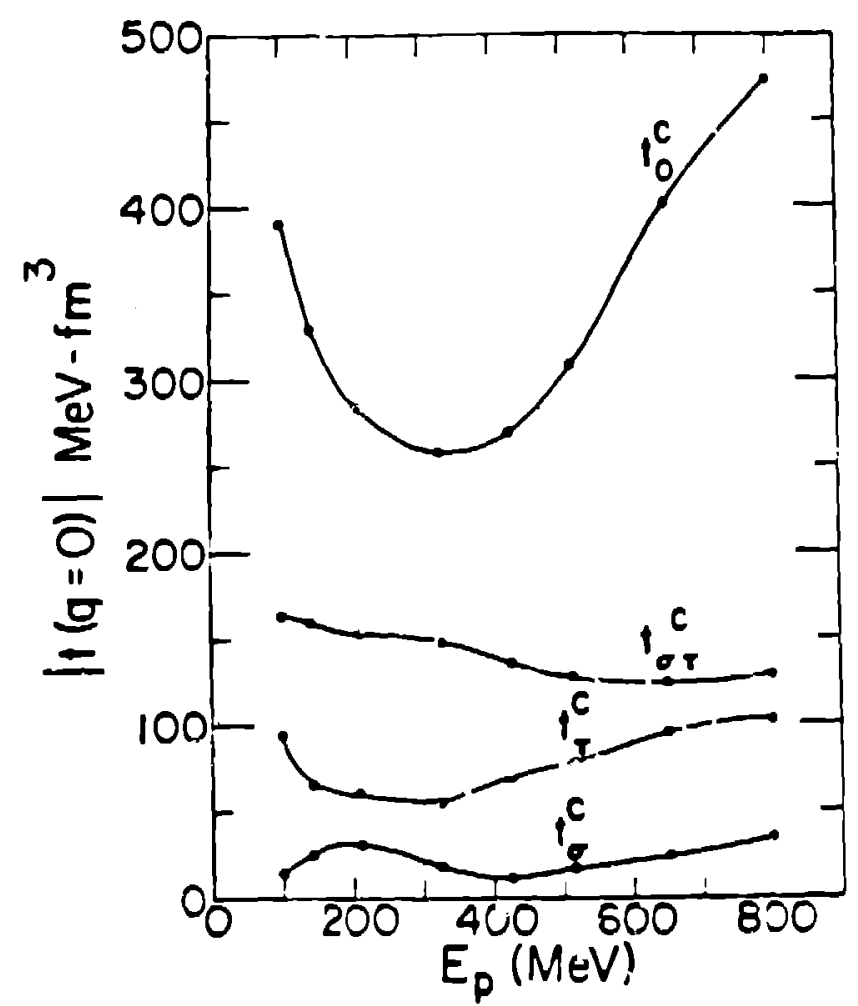

Fig. 2 Energy dependence of the $N-N$ t matrix at $q=0$ for various spin and isospin channels.

Finally on the subject of theoretical developments I want to mention the work of Amado, Lenz, MeNe11, and SFarrow (ALMS, ${ }^{\circ}$ who have derivad a set of very simple "data-data" relations, and who have, in the process, considerably clarified the physics of elastic s attering and inelastic scattering to collective states at intermediate energles. Starting from the eikonal $\mathrm{N}$-nucleus scattering amplitude aild employing the Tassie model for transition densities, ALMS are able to show that the cross section for inelastic scattering to a state of spir: $L$ (natural parity) is given by

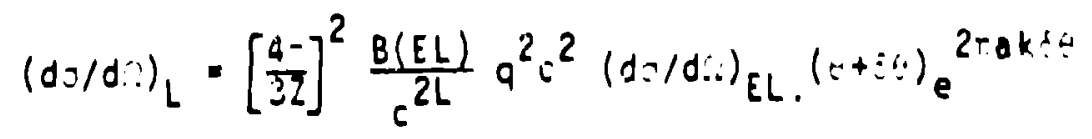

wit.h

$$
\begin{aligned}
& d \theta=\frac{(L-1)}{k c}: \text { for } L \text { odd } \\
& \delta \theta=\frac{(L-1) t+-/ 2}{k c} \text { for } L \text { even }
\end{aligned}
$$

and

$$
+\tan ^{-1}(\pi a / c)
$$

where a and $c$ are respectively the diffuseness and half-density radius of a Fermi distribution. The remarkable feature of this equation is that it bypasses the thaoretical description of elastic scattering usually required for inelastic scattering - only experimental data are used. As ALMS have

$-3$. 
pointed out, the data-to-data feature nas the effect of removing some of the inaccuracies present in the orginal theory. For example, imperfect optical model fits result in imperfect inelastic cross section predictions.

This model has been extended to describe polarization observabies as well as crnss sections. It is clear from the excellent agreement obtained with experimental analyzing power ( $F$ ig. 3 ) that this observable contributes no new nuclear structure information for most natural parity transitions i $t$ high energies.

\subsection{Gtant Resonances}

3. Nuclear Structure and Reactions

Intermediate-energy proton and light-iun scattering is an ideal tool for studying one of the most foundamental characteristics of nuclear spectra giant resonances (GR). It is surprising then that unt il recently relativei. itttle inpus into this important subf ield of nuclear physics had come from medium energy facilities. This situation is rapidly changing, however, as should be apparent in this talk.

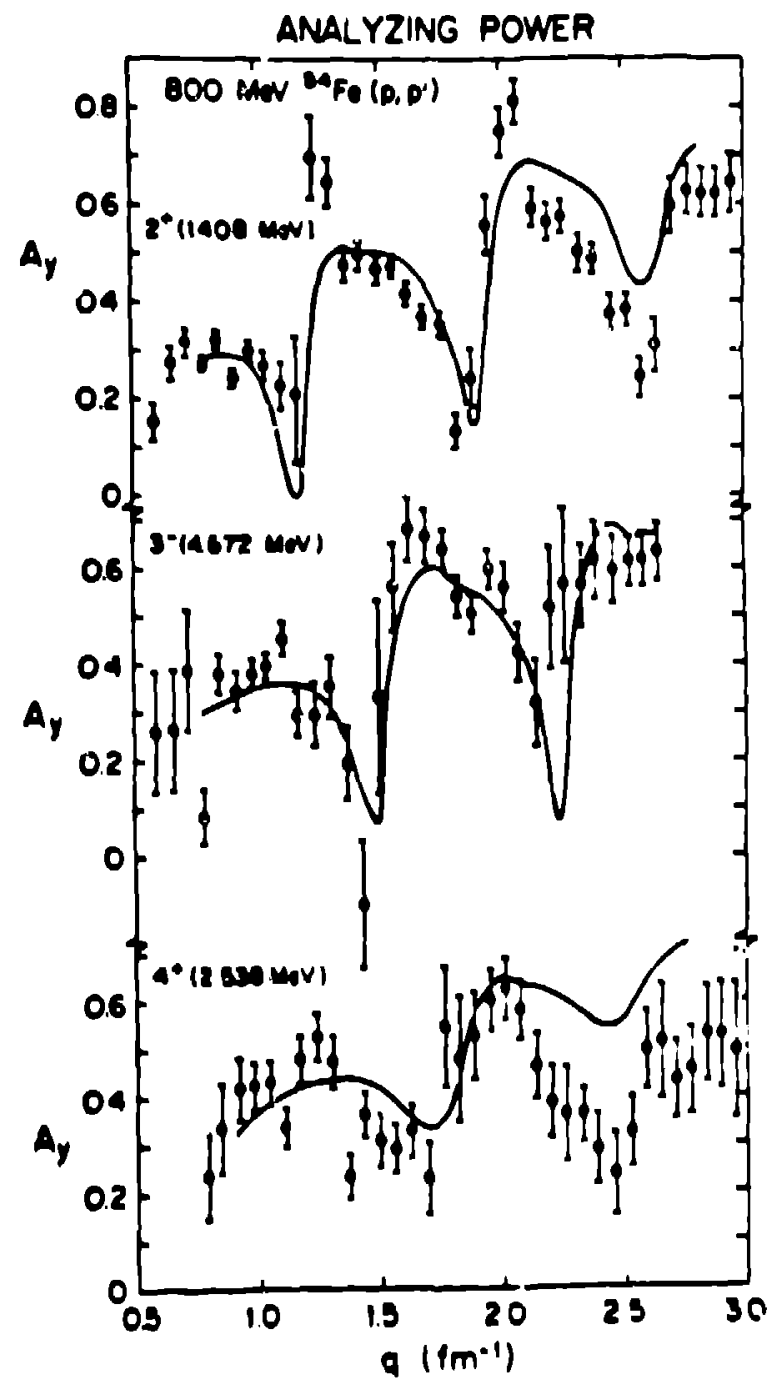

The first observation of GR excitation at high-energy was made at Saclay where $1.37 \mathrm{GeV}$ aipha particles were used to excite the giant quadrupole resonance $(G Q R)$ in $30 \mathrm{Ni} . \mathrm{A}^{3}$ It is encouraging to see this work continuing at $S d c$ lay with $480 \mathrm{MeV}$ alphas."

The first new GR observed at on intermed tate-energy facility was the high-energy oc:upole giant resonance (HEOR) at $E_{x} 2110 / \mathrm{A} / 1 / 3 \mathrm{MeV}$, observed by our group at LOS Alamos with $800 \mathrm{MeV}$ proten scatterirg's (Fig. 4). This isoscalar rescnance was predicted theoretically, 16 but not observed in numerous studies with 100 to $150 \mathrm{MeV}$ alpha particles due to backgrounds arising from complex reaction Diocesses (e.g. ("He, "He*)) which obscure the high excitation energy region. High-energy protons appear to have the most favorable resonance-tocontinuum background ratio yet observed in any $G$ P experiment. This has allowed us to study the systenatics ( $F(g .5)$ of the HEOR over a wide range of $A$, including the relatively light nuclet "Ca and $3{ }^{\prime} 1$. where the resonance is 8 to $10 \mathrm{MeV}$ wide.

Fig. 3 Data-to-Data calculations of tile andlyzing powers of coliective transitions. 


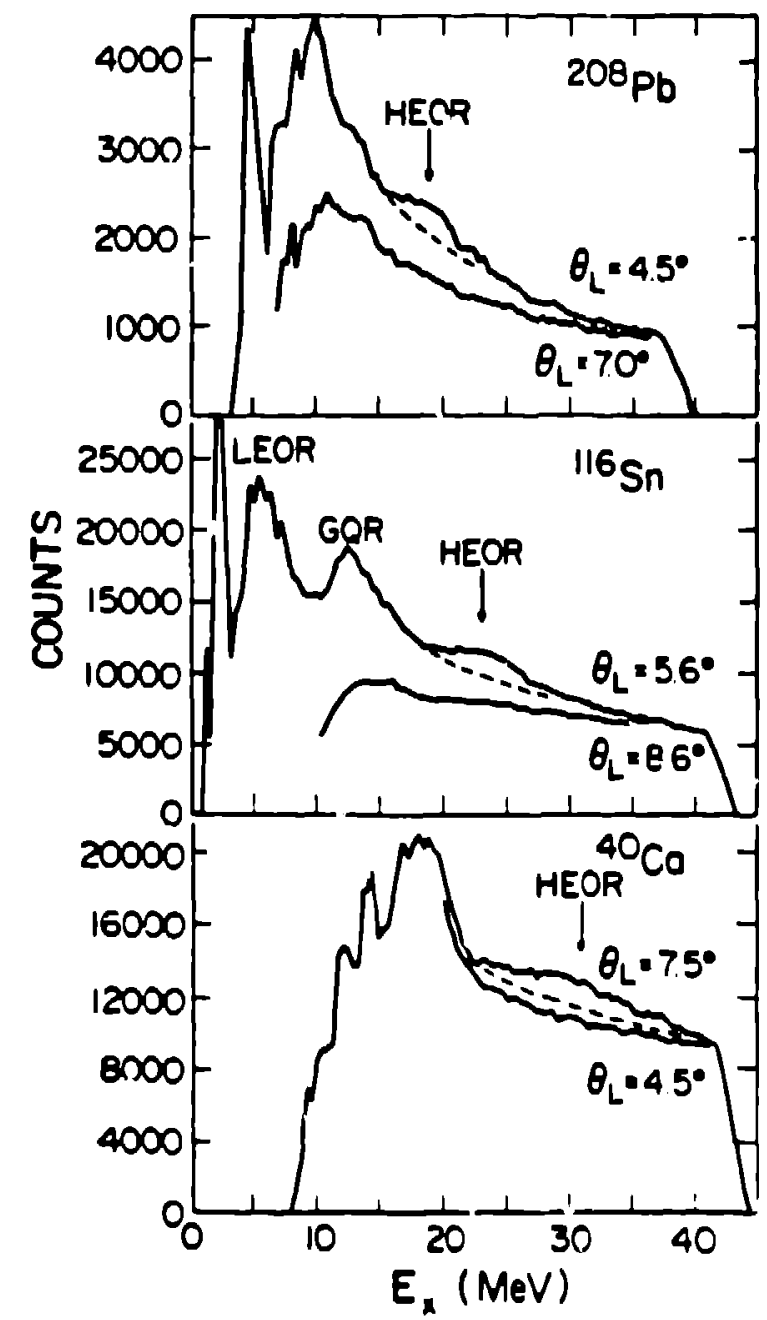

Analysis of the strength of scalar-isoscalar GR's is commonly made in terms of the energy-weighted sum rule (EWSR)

$$
\sum_{n} E_{n} B(E \ell)_{n}=\left(\frac{\hbar^{2} A}{8 \pi m}\right) \ell(2 \theta+1)\left\langle r^{2 \ell-2}\right\rangle
$$

which is a nearly model-independent quantity. Theuretical calculations indicate about 40 to $50 \%$ of the isoscalar E 3 EWSR concentrated in the HEOR.16 Our data show only $25+\frac{j}{0}$. Whether or not this is a real discrepancy depends critically on the assumption one makes about the continuum background. At present we use the standard and completely arbitrary procedure of drawing a reasonable line (dashed ine in Fig. 4) and subtracting it from the data. It may soon be possible to improve on this method substantially. The continuum underlying GR's appears to be largely quasi-elastic scattering. Muitiple. scattering theory calculations of this process $"$ hold promise for a quantitative description of small a continuum spectra.

Fig. 4 Specti from 800 ilev proton inelastic scattering snowing the nigh-energy or wupole glant resonance (HEOR). The lower spectrum for each target corresfonds to a minimum in the octupole angular distribution.

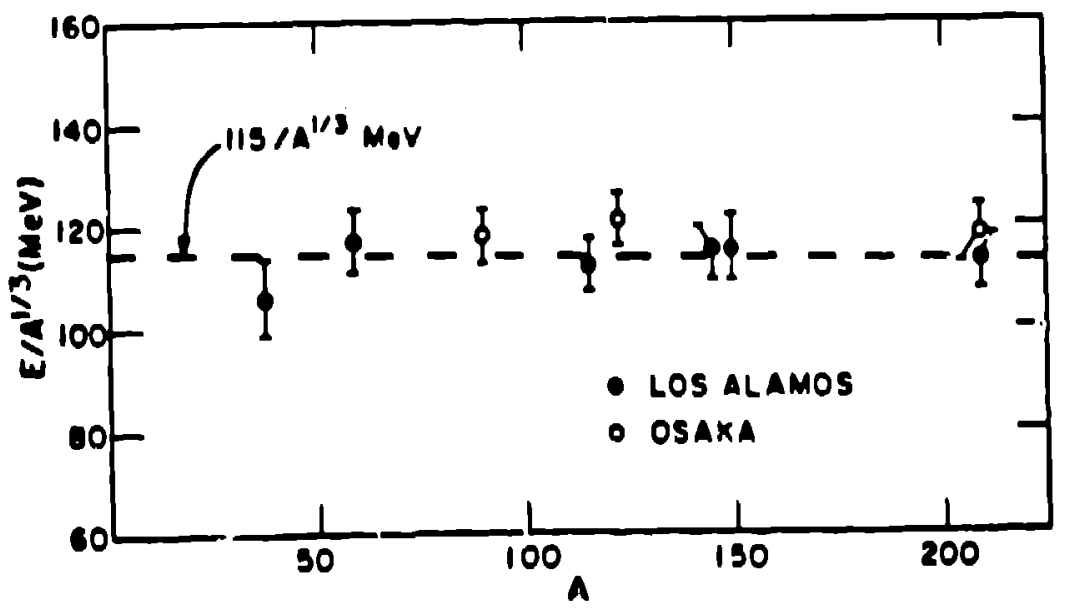

Fig. 5. Excitation energy systematics of the high energy octupole glant resonance. 
With the discovery of the HEOR it is natural to ask, "are there GR's of nigher multipolarity?" Our answer to this is a tentative, "no". In II sn, the most completely studied case, we have taken very high statistics spectra in the region of the maximum for $\ell=5$ and found only featureless continuum. Studies of $l=4$ sirength in the vicinity of the GQR ${ }^{1}$ likewise indicate no great concentration of strength. Thus, at present, the possibility of finding well-defined GR's with $\ell>3$ seems remote.

To finish the discussion of GR's I want to briefly mention a new and very exciting discovery made at Orsay'9. Figure 6 shows $200 \mathrm{MeV}\left(p, p^{\prime}\right)$ spectra on the $2 r$ isotopes at very small angles. The peak near $E_{x}=8 \mathrm{MeV}$ has an angular

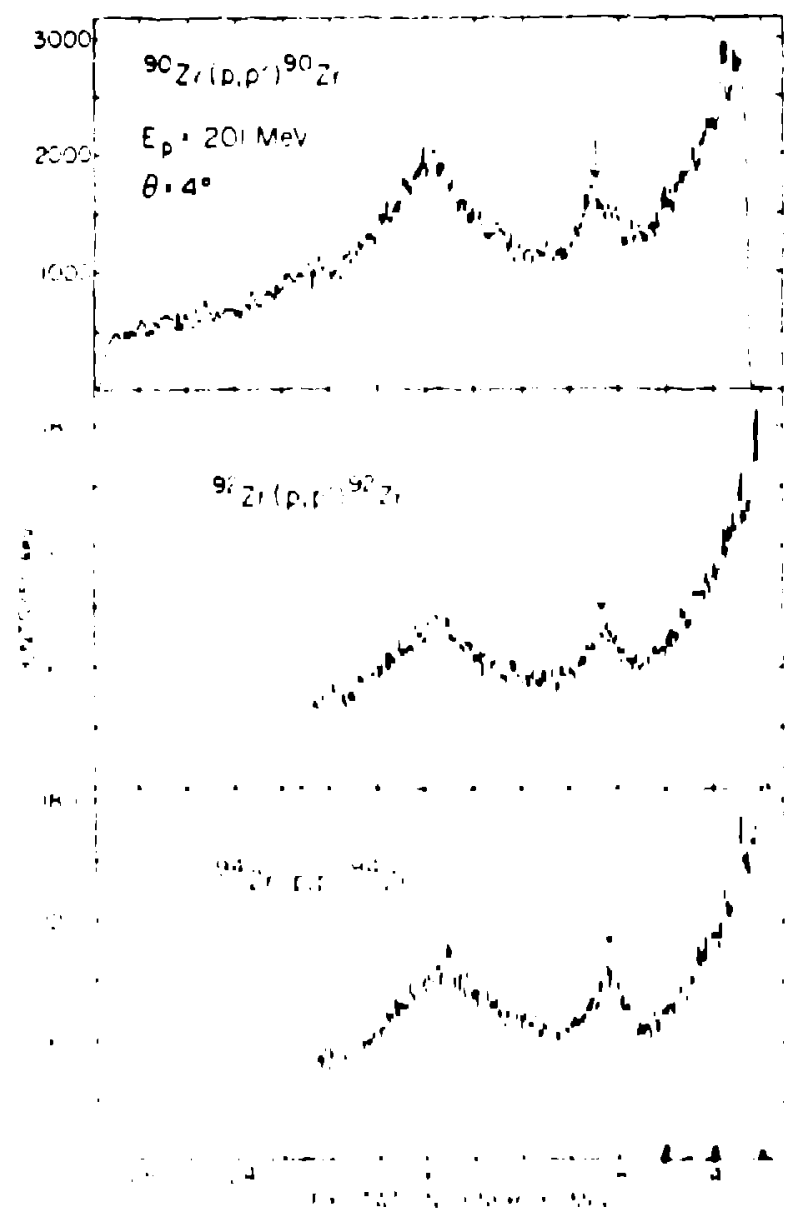

Fig. 6 spectra from 200 kaV proton inelastic scattering snowing the excltatiun of the Ml resonances (Indicated by arrows).

distribution characteristic of $\ell=0$. Since we know that the giant monopole resonance near $E_{X} \sim 16 \mathrm{MeV}$ contains most of the $P .0(A S=0)$ strength, the most logical explanation is that the 8 MeV peak is the glant $M$ l resonance ( $\left.\Delta l=0, \Delta s=1, \Delta^{T}=1\right)$. Its strength is about half of the single-particie sum rule, in rough agreement with the systematics of the Gamow Teller (GT) reconance. 20 The retardation of the strengths of the $M$ l and GT resonances has acquiret increased significance as new theorctical evidenceal suggests that adinixtures of delta isobar-nole conflgurations may provide the answer.

\subsection{PION - COINDENSATION PRECURSORS}

A flurry of interest was generated about three years ago by the posstollity thai norinal nuclear matter might be suffictent ly close to the critical density for plon condensation to show evidence of precritical 
behavior. Scattering of electrons and protons at momentum transfers of 22 $m_{\pi}$ to $3 m_{\pi}$ was suggested 2,23 as the most likely ground for observing possible precritical enhancements in the pion field of nuclei. Electrons, of course, offer the advantage of a well-known interaction. Their disadvantage is that they don't couple directly to pions, with the effect that (e,e') from a slab of near-critical nuclear matter would show no enhancement (for finite nuclei (e,e') may still probe the change in the pion field in the nuclear surface). Protons couple directly to pions but are, unfortunately, strongly interacting particles and therefore subject to another set of uncertainties. Before describing the results of the experiments which are simple. I want to make a brief remark about the theories which are not simple. A crucial quantity in any calculation of pion condensation or precritical enhancement is the Landau-Migdal paramter $g^{\prime}$, which is a measure of the effect of short range correlations. When $g^{\prime}$ is assumed to be small $\left(g^{\prime} \sim .3\right)$, the density for pion condensation is low, and predicted precritical enhancements are large. Conversely values of $g^{\prime}$ around 0.7 produce much higher critical densities and the corrisponding enhancement in large a form factors is small.

A number of ( $\left.p, p^{\prime}\right)$ experiments ${ }^{2}, 2 s^{2}$ with energies ranging from 122 to $300 \mathrm{MeV}$ have searched for large q enhancements in the cross section of the $1^{+}$, $T=1,15.11 \mathrm{MeV}$ state of ${ }^{12} \mathrm{C}$. Figure 7 shows the combined data from two such experiments performed at Saturne and LAMPF. These experiments are probably

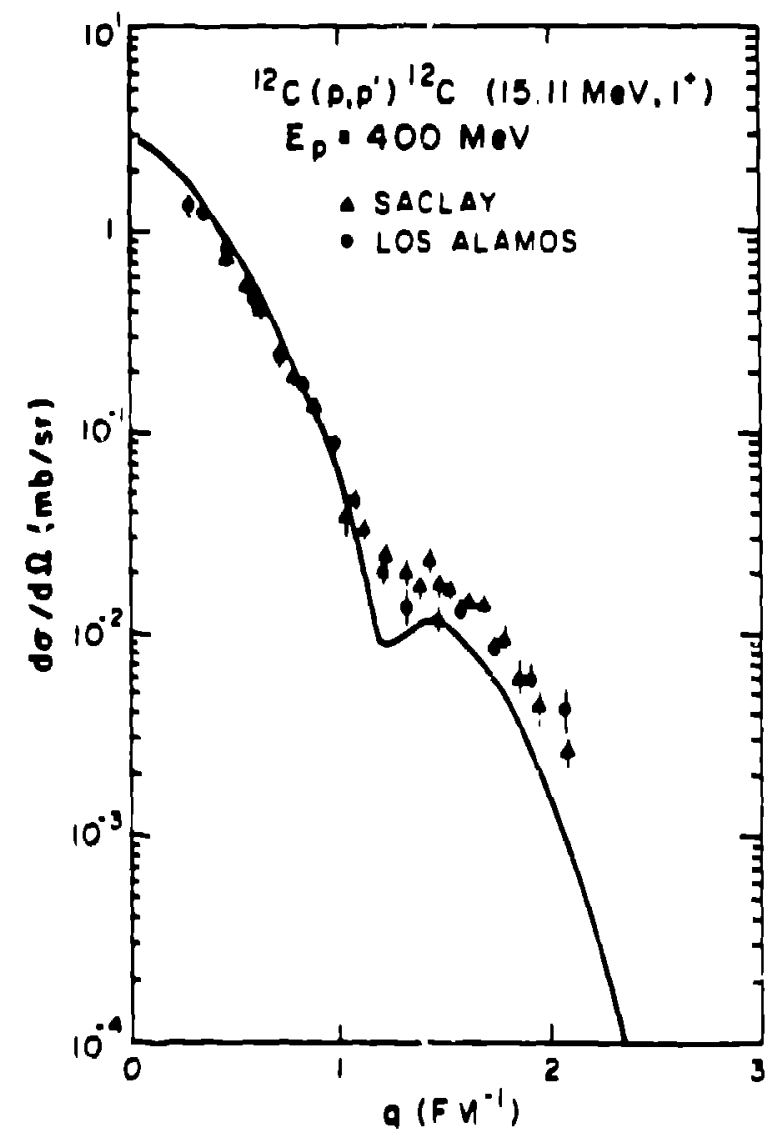

F1g. 7 Angular distribution for the excitation of the $15.11 \mathrm{MeV}$ state in $12 \mathrm{C}$ with $400 \mathrm{MeV}$ protons. The curve is aWIA calculation which is described in the text. 
the best test of precritical enhancement thus far because the beam energy is high enough for the impulse approximation to be valid, and low enough so that the $N-N$ interaction is known. The solid curve is a DWIA calculation by Love and Franey ${ }^{26}$ using the Cohen-Kurath ${ }^{7}$ wave functions. This calculation can safely be sald to contain no precritical enhancement. An ennancement such as would result, rom a value of $g^{\prime} \sim 0.5$ would raise the cross section in the $q=$ 2 to $3 \mathrm{~m}_{\pi}$ region by pernaps a fictor of 10 . Although there is some discrepancy in the direction of precri:icai enhancement, several other non-exotic improvements in the nuclear wavi: functions need to be taken into account ${ }^{20}$ before a quantitative analysis in terms of $g^{\prime} c a n$ be made.

The status of all present evidence, from $\left(p, p^{\prime}\right),\left(e, e^{\prime}\right)$, and the analys is of unnatural parity states, ${ }^{2}{ }^{2}$ is that $g^{\prime}>0.65$ and does not strongly depend on $q$. Thus nuclei are not very close to the critical density for pion condensation and precritical ennancements are probably unobservably small.

\subsection{New Polarization Experiments}

\subsubsection{General}

One of the most attractive features of high-energy protons is that their long range makes possible the design of very efficient polarimeters. This has the consequence that the triple-scattering observables may be measured for nearly any reaction for which cross sections and analyzing powers can be measured. Two programs to exploit this new physics are underway, one using the QDOM spectrometer and a very simple focal-plane polarimeter for 150 to 200 Me: protons at the Indlana University Cyclotron Facility (IUCF), 30 and the other using a very extensive mult 1 -wire chamber configuration ( $F i g .8$ ) in the $f o c a$ ) plane of the nigh-resolution-spectrometer (HRS) at LAMPF. The latter utilizes the energy range 300 to $800 \mathrm{MeV}$. Initial work with the IUCF polarmeter is described in a contribution at this conference. I will briefly describe the experiments on the HRS polarimeter, which has been in operation for about 9 months (See the Contribution by J. F. Amman at this conference).

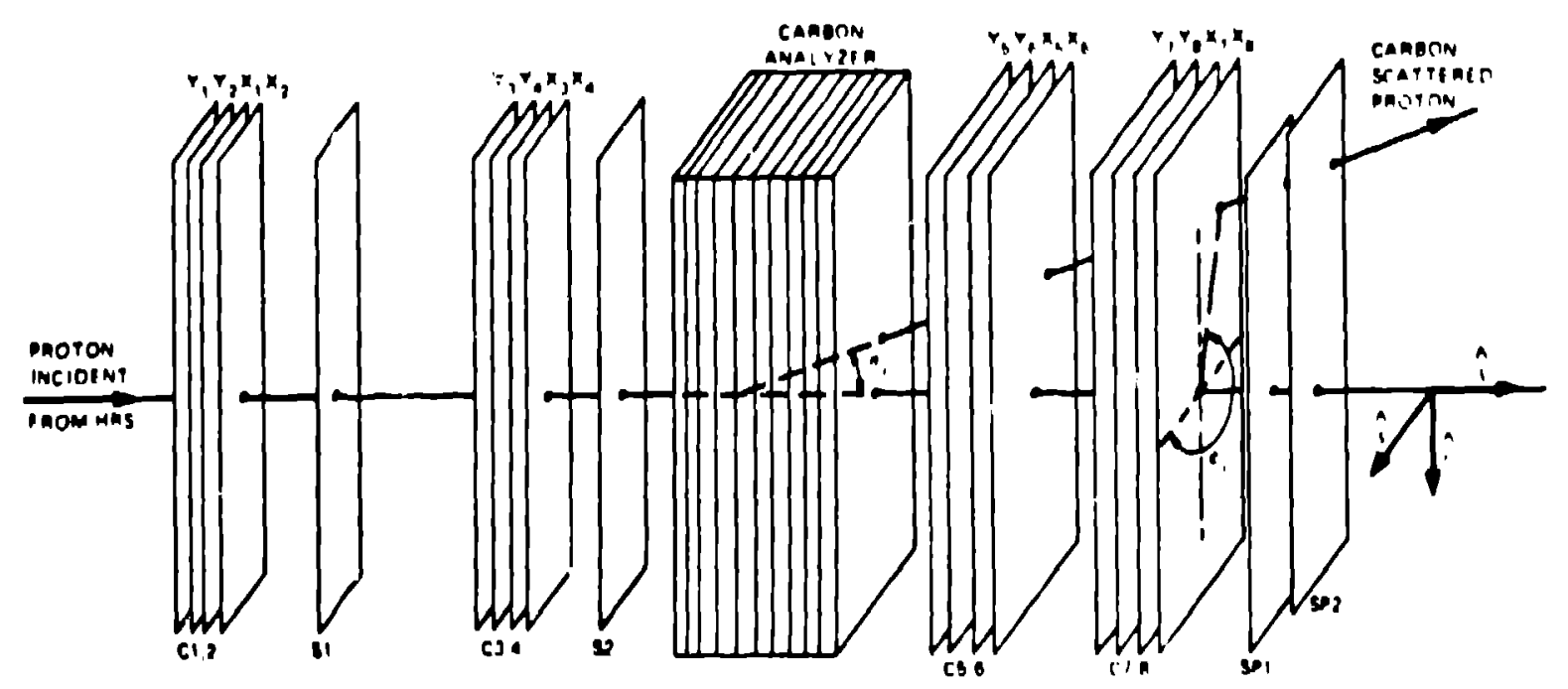

F tg. 8 Schematic representation of the HRS focal plane polarimeter. $X$ and $Y$ denote multiwile drift chambers with position sensitivity in the vertical and norlzontal planes, respectively. S and SP denote scintillators. 
The HRS polarimeter is an extremely versatile device which utilizes an array of multi-wire chambers to reconstruct the trajectory of protons scattered in a carbon block, as well as to perform the usual functions of determining the momenta and scattering angles from the primary scattering. The data acquisition system employs a fast microprocessor to reject events not scattered in the carbon block. Because of the orientation of the magnetic field of the HRS, the component of spin sideways and in the reaction plane (s) does not precess. The components normal $(n)$ to the reaction plane and longitudinal ( $l$ ) precess by about $296 \gamma$ degrees ( $\gamma$ is the Lorentz factor) and are not measureable for certain values of the outgoing momentum. The pclarization from the LAMPF accelerator may be adjusted to yield $s, n$, or $l$ initial polarization, thus any of the Wolfenstein triple scattering parameters ${ }^{31}$ may be measured (with the exception mentioned above).

\subsubsection{Measurement of $Q$ fo, Elastic Scattering}

The scattering amplitufe for a spin $1 / 2$ projectile on a spin zero nucleus can be expressed as

$$
F(q)=g(q)+h(q) \sigma \cdot n
$$

The usual cross section and analyzing powers give

$$
\begin{aligned}
& d \sigma / d \Omega=|g|^{2}+|n|^{2} \\
& A(d \sigma / d \Omega)=2 \operatorname{Re}\left[g h^{\star}\right]
\end{aligned}
$$

By combining the triple scattering parameters, $R$ and $A$, a new and complementary quantity $Q$ can be constructed

$$
Q(d \sigma / d \Omega)=2 \operatorname{Im}\left[g h^{\star}\right]
$$

The measurement of $d \sigma / d \Omega, A$, ind $Q$ results in a complete determination of $F(0)$ apart from an overail prase.

The results of the first measurements of the $Q$ parameter are shown together with the analyzing power (or polarization $p$ for elastic scattering) in figure 9. This solid curve is a Glauber model calculation using the methods of Bleszynski and Osland ${ }^{32}$ (The other curves are aiscussed in ref. 33 ). It is apparent that neither observable is quant itatively reproduced by the calculation in spite of the fact that at $E_{p}=500 \mathrm{MeV}$ the $N-N$ phase shifts are well known. Obviously future measurements of new observables such as $Q$ will present riew challenges to the theories of hadron-nucleus scattering.

\subsubsection{Spin-flip}

The transverse spin-flip probability (SFP), $S$, is an observable winich is closely connected to spin transfer ( $\Delta s=1)$ in elastic or inelastic-scattering. In terms of the wolfenste in parameter, $0, S=1 / 2(1-0)$. In general it can be shown that $S>0.5$ when $\Delta s=1$ dominates a reaction and $S$ 20 when $\Delta s=0$ is dominant. ${ }^{44}$ The nope is that the SFP can be used as a signature of spin transfer processes in proton scattering and thereby probe such interesting nuclear structure phenomena as collective spin excitation and perhaps elucidate the validity of theoretical models of reactions where the tensor and spin-spin interactions are important. 


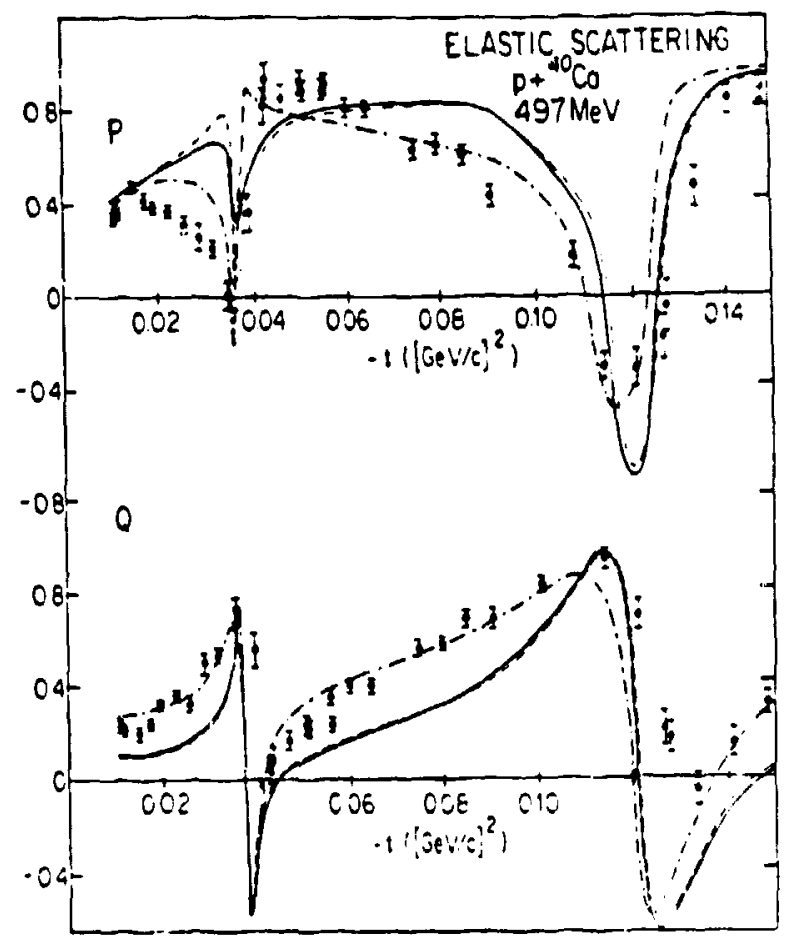

Fig. 9 Analyzing power and the $Q$ parameter for elastic scattering from ${ }^{40} \mathrm{Ca}$. The solid curve is a Glauber model calculation described in the text.

The latter objective is the primary aim in the first investigations (at LAMPF and IUCF) of the SFP at intermediate energies. Figure 10 shows the data for the two $\mathrm{I}^{+}$states of ${ }^{12} \mathrm{C}$ excited by $400 \mathrm{MeV}$ protons. Since $\Delta S=1$ excitation must occur if a single-step scattering dominates (an assumption whiin needs verification) one expects the SFP to be large. Quantitatively this is seen in the data and corifirmed by DWIA calculations using the Love-Franey interaction and the Conen-Kurath wave functions. Some divergence between theory and experiment is seen for the $15.11 \mathrm{MeV}$ state at the larger momentum transfers. The reason(s) for this is (are) nut known at present, nor does one understand why the SFP for the $1^{+} T=0$ state is reasonably well described by the DWIA whereas do/d $\Omega$ is not. The next couple of years should provide a much larger base of spin-flip and related experiments and eventually resuit in an increased understanding of the complex and rich physics of proton-nuc leus scattering.

\section{Conclusion}

I hope that I have been able to convince you that high-energy proton light-ion scattering has recently made some very significant contributions to nuclear structure physics. Some of the areas touched on - giant resonances, possible evidence for delta isobar-hole configurations, and pion condensation precursors-have broad implications for many areas of nuclear physir.s. It should be obvious that new experimeital methods (eg. polarimeters) combined with increasingly powerful theoretical techniques will make the next few years of high-energy proton and light-ion physics even more exciting. 


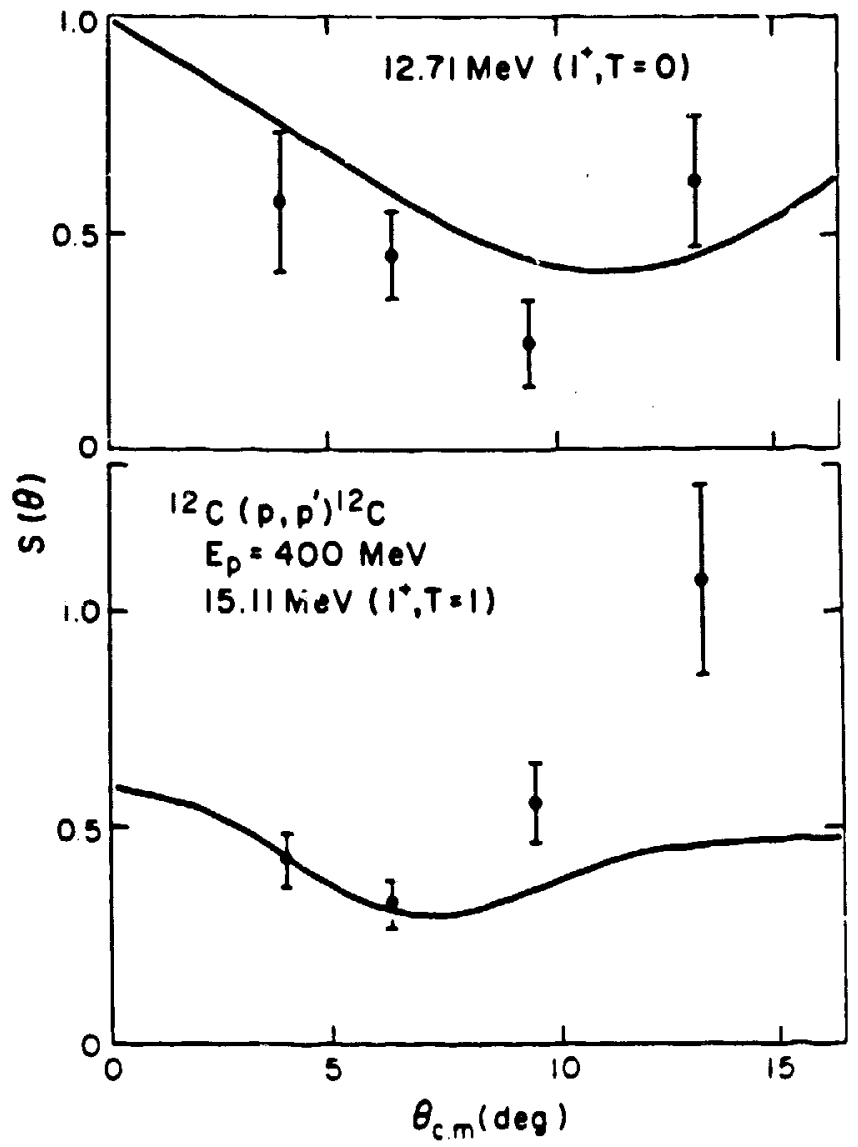

Fig. 10 Spin flip probabilities for the $i^{+}$states of ${ }^{12} \mathrm{C}$ with 400 l:eV protons. The curves are DWIA calcu'ations described in the text.

\section{References}

1) C. A. Whitten, Proc VIIIth. Int. Conf. On High Energy Physics and Nuclear Structu: $\therefore$ Vancouver, 1979, ed. D. F. Measday and A. W. Thomas (North Holland Publishing Company - Amsterdam) P419, and references therein.

2) F. Petrovich and W. G. Love, Proc. I t. Conf. On Nucleir Finysics, Berkeley Calif., 1980, ed. R. M. Diamond and J. O. Rasmussen (North Hol? and Publisning Co. - Amsterdam).

3) G. W. Hoffmann, et al. (submitted to Phys. Rev. C.).

4) For a recent review of this subject see L. Ray, Proc. Vth Int. Symposium on Polarization Phenomena in Nuclear Physics, Santa Fe, NM, 1980, ed. G. G. Onlsen, et al. (American Inst. of Physics, New York) $\mathrm{P} .255$.

5) A. K. Kerman, H. McManus, and R. M. Thaler Ann. Phys. 8 (1959) 551; R. J. Glauber, in Lectures in Theoretical Physics, ed. W. E. Brittin, Vol. i, (Interscience, New York, 1959).

6) S. J. Wallace, Ann. Rev of Nucl. Sci. (to be published).

7) See contributions in the session on Nucleon-Nucleon Polarization Phenomena, Proc. Vth int. Symposium on Polarization Phenomena in Nuclear Physics, Santa Fe, NM, 1980, ed. G. G. Ohlsen et al. (American Inst. of Physics, Ne.: York).

8) R. A. Arndt, Bull. Am. Phys. Soc. 26, (1921) 528.

9) W. G. Love and M. A. Franey (Phys. Rev. $C$, in press); W. G. Love, The $(p, n)$ Reaction and the Nucleon-Nucleon Force, eds. C. D. Goodman, et al TPlenum, New York, 1980) p. 23. 
10) R. D. Amado, F. Lenz, J. A. McNeil, and D. A. Sparrow, Phys. Rev. C22, (i980) 2147; J. A. MCHeil and D. A. Sparrow, Priys. Rev. C23 (195i) 2TL4.

11) J. R. Comfort, et al., Phys. Rev. C21, (198,0) 21471

12) J. Kelly, et al., Phys. Rev. Lett. 45, (1930) 2012.

13) T. S. Baver, et al., Phys. Rev Cl9 T979) 1438.14)B. Bonin, el al., Proc. Int. Conf. on iluclear Physics, Berkeley, ed. R. M. Uiamond and J. 0. Rasmussen (North Holland - Amsterdam).

14) B. Bonin, et al., Proc. Int. Conf. un Nuclear Physics, Berkeley, ed. R. M. Diamond and J. O. Rasmussen (1vorth Holland - Amsterdami).

15) T. A. Carey, et al., Phys. Rev. Lett. 45 (1980) 239.

16) G. F. Bertsch and S. F. Tsai, Phys. Rep. 18C (1975) 127, K. F. Liu and G. E. Brown, Nucl. Phys. A<65 (1976) 385.

17) G. F. Bertsch and C. Schotten - private communication.

18) F. E. Betrand, et al., (to be publisned).

19) N. Ananiaraman, et al., Phys Rev. Lett. 46, (1981) 1318.

20) C. D. Goodman, et al., Phys. Rev. Lett. $\overline{44}(1980) 1755$.

21) E. Oset and $M$. Rno, Phys. Rev. Lett. 42 T979) 47; G. Bertsch, Proc. Int. Conf. On Nuclear Pnysics, Berkeley, 1980 en., R. M. Diariond and J. 0.

Rasmussen (North Holland - Amsterdam) P. 157C; A. Bonr and i. R. Mottelson, Pnj's. Lett. 10013 (1981) 10.

22) M. Ericson and J. Delorme, Fnys. Lett. 763 (1975) 18C; J. Delurme et al., Phys. Lett. 893 (1980) 327.

23) H. Toki and W. Wei.e, Pnys. Rev. Lett. 4i (19\%) 1034.

24) J. Comfort, et al., Phys. Rev [.23, (198T) 1358.

25) M. Haji - Saeid et al., Pnys. Rev. Lett 45 (1980) 380.

26) W. G. Love and M. Franey - private communication.

27) S. Conen and D. Kurath, Nucl. Phys. 73, (1965) 1.

28) See discussions in H. Toki and W. Weise, Phys. Le'.t. Gelf $(1950) 265$.

(9) J. Meyer -ter-Venn (to be published).

30) T. A. Carey, et al. - contribution to this conference.

31) L. Wolfenstein, Ann. Rev. Nucl. Sci. $5(1956) 4 j$.

32) M. Bleszynsk: and P. Osland, Phys. Lett. 84l. (1079) 15?.

33) A. Ranoar, et al., Los Alamos Nat. Lab. Report LA-JR-S1-165) (sub-ittej to Phys. Rev. Lett.)

34) W. D. Comelius, J. M. Moss, and T, Yaniaya, Pnys. Rev. Czi, (10s1) 130i... 